\title{
Some New Results on Prime Graphs
}

\author{
Samir K. Vaidya ${ }^{1}$, Udayan M. Prajapati ${ }^{2}$ \\ ${ }^{1}$ Saurashtra University, Rajkot, India \\ ${ }^{2}$ St. Xavier's College, Ahmedabad, India \\ Email: samirkvaidya@yahoo.co.in, udayan64@yahoo.com
}

Received May 6, 2012; revised June 13, 2012; accepted June 25, 2012

\begin{abstract}
We investigate prime labeling for some graphs resulted by identifying any two vertices of some graphs. We also introduce the concept of strongly prime graph and prove that the graphs $C_{n}, P_{n}$, and $K_{1, n}$ are strongly prime graphs. Moreover we prove that $W_{n}$ is a strongly prime graph for every even integer $n \geq 4$.
\end{abstract}

Keywords: Prime Labeling; Prime Graph; Strongly Prime Graph

\section{Introduction}

We begin with finite, undirected and non-trivial graph $G=(V(G), E(G))$ with the vertex set $V(G)$ and the edge set $E(G)$. Throughout this work $C_{n}$ denotes the cycle with $n$ vertices and $P_{n}$ denotes the path on $n$ vertices. In the wheel $W_{n}=C_{n}+K_{1}$ the vertex corresponding to $K_{1}$ is called the apex vertex and the vertices corresponding to $C_{n}$ are called the rim vertices, where $n \geq 3$. The star $K_{1, n}$ is a graph with one vertex of degree $n$ called apex and $n$ vertices of degree one called pendant vertices. Throughout this paper $|V(G)|$ and $|E(G)|$ denote the cardinality of vertex set and edge set respectively.

For various graph theoretic notation and terminology we follow Gross and Yellen [1] while for number theory we follow Burton [2]. We will give brief summary of definitions and other information which are useful for the present investigations.

Definition 1.1: If the vertices are assigned values subject to certain condition(s) then it is known as graph labeling.

Vast amount of literature is available in printed as well as in electronic form on different kind of graph labeling problems. For a dynamic survey of graph labeling problems along with extensive bibliography we refer to Gallian [3].

Definition 1.2: A prime labeling of a graph $G$ is an injective function $f: V(G) \rightarrow\{1,2,3, \cdots,|V(G)|\}$ such that for every pair of adjacent vertices $u$ and $v$, $\operatorname{gcd}(f(u), f(v))=1$. The graph which admits a prime labeling is called a prime graph.

The notion of a prime labeling was originated by Entringer and was discussed in a paper by Tout et al. [4]. Many researchers have studied prime graphs. For e.g. Fu and Huang [5] have proved that $P_{n}$ and $K_{1, n}$ are prime graphs. Lee et al. [6] have proved that $W_{n}$ is a prime graph if and only if $n$ is even. Deretsky et al. [7] have proved that $C_{n}$ is a prime graph.

Definition 1.3: Let $u$ and $v$ be two distinct vertices of a graph $G$. A new graph $G_{u, v}$ is constructed by identifying (fusing) two vertices $u$ and $v$ by a single new vertex $x$ such that every edge which was incident with either $u$ or $v$ in $G$ is now incident with $x$ in $G_{u, v}$.

Vaidya and Kanani [8] have established that the graph obtained by identifying any two vertices $u$ and $v$ (with $d(u, v) \geq 3)$ of $C_{n}(n \geq 4)$ is a prime graph. The switching invariance of various prime graphs is discussed by Vaidya and Prajapati [9]. In the present paper we investigate further results on prime graphs.

Bertrand's Postulate 1.4: For every positive integer $n>1$ there is a prime $p$ such that $n<p<2 n$.

\section{Prime Labeling of Some Graphs}

Theorem 2.1: The graph obtained by identifying any two vertices of $K_{1, n}$ is a prime graph.

Proof: The result is obvious for $n=1,2$. Therefore we start with $n \geq 3$. Let $v_{0}$ be the apex vertex and $v_{1}, v_{2}, \cdots, v_{n}$ be the consecutive pendant vertices of $K_{1, n}$. Due to the nature of $K_{1, n}$ two vertices can be identified in following two possible ways:

Case 1: The apex vertex $v_{0}$ is identified with any of the pendant vertices (say $v_{1}$ ). Let the new vertex be $u_{0}$ and the resultant graph be $G$.

Then $\operatorname{deg}\left(v_{i}\right)=1$, for $i=2,3, \cdots, n$ and $\operatorname{deg}\left(u_{0}\right)=n+1$ as there is a loop incident at $u_{0}$. Define $f: V(G) \rightarrow\{1,2, \cdots, n\}$ as $f\left(v_{i}\right)=i$ for $i=2,3, \cdots, n$ and $f\left(u_{0}\right)=1$. Obviously $f$ is an injection and $\operatorname{gcd}(f(u), f(v))=1$ for every pair of adjacent vertices 
$u$ and $v$ of $G$. Hence $G$ is a prime graph.

Case 2: Any two of the pendant vertices (say $v_{n-1}$ and $v_{n}$ ) are identified. Let the new vertex be $u_{n-1}$ and the resultant graph be $G$. So in $G, \operatorname{deg}\left(v_{i}\right)=1$, for $i=1,2$, $\cdots, n-2, \operatorname{deg}\left(u_{n-1}\right)=2$ and $\operatorname{deg}\left(v_{0}\right)=n$. Define $f: V(G) \rightarrow\{1,2, \cdots, n\}$ as $f\left(v_{i}\right)=i+1$ for $i=0,1,2$, $\cdots, n-2$ and $f\left(u_{n-1}\right)=n$. Obviously $f$ is an injection and $\operatorname{gcd}(f(u), f(v))=1$ for every pair of adjacent vertices $u$ and $v$ of $G$. Hence $G$ is a prime graph.

Illustration 2.2: The prime labeling of the graph obtained by identifying the apex vertex with a pendant vertex of $K_{1,7}$ is shown in Figure 1.

Illustration 2.3: The prime labeling of the graph obtained by identifying two of the pendant vertices of $K_{1,7}$ is shown in Figure 2.

Theorem 2.4: If $p$ is a prime and $G$ is a prime graph of order $p$ then the graph obtained by identifying two vertices with label 1 and $p$ is also a prime graph.

Proof: Let $f$ be a prime labeling of $G$ and $i$ be the label of the vertex $v_{i}$ for $i=1,2, \cdots, p$. Moreover $u_{1}$ be the new vertex of the graph $G_{1}$ which is obtained by identifying $v_{1}$ and $v_{p}$ of $G$. Define

$$
\begin{aligned}
& f_{1}:\left\{u_{1}, v_{2}, v_{3}, \cdots, v_{p-1}\right\} \rightarrow\{1,2, \cdots, p-1\} \text { as } \\
& f_{1}(x)= \begin{cases}f\left(v_{i}\right) & \text { if } x=v_{i}, i=2,3, \cdots, p-1 \\
1 & \text { if } x=u_{1} .\end{cases} \\
& \text { Then } f_{1}(x)= \begin{cases}i & \text { if } x=v_{i}, i=2,3, \cdots, p-1 \\
1 & \text { if } x=u_{1} .\end{cases}
\end{aligned}
$$

Obviously $f_{1}$ is an injection. For an arbitrary edge $e=u v$ of $G_{1}$ we claim that $\operatorname{gcd}\left(f_{1}(u), f_{1}(v)\right)=1$. To prove our claim the following cases are to be considered.

Case 1: If $u=u_{1}$ then $\operatorname{gcd}\left(f_{1}(u), f_{1}(v)\right)=$ $\operatorname{gcd}\left(f_{1}\left(u_{1}\right), f_{1}(v)\right)=\operatorname{gcd}\left(1, f_{1}(v)\right)=1$.

Case 2: If $u \neq u_{1}$ and $v=u_{1}$ then $\operatorname{gcd}\left(f_{1}(u), f_{1}(v)\right)$ $=\operatorname{gcd}\left(f_{1}(u), f_{1}\left(u_{1}\right)\right)=\operatorname{gcd}\left(f_{1}(u), 1\right)=1$.

Case 3: If $u \neq u_{1}$ and $v \neq u_{1}$ then $u=v_{i}, v=v_{j}$ for some $i, j=2,3, \cdots, p-1$ with $i \neq j$ then $\operatorname{gcd}\left(f_{1}(u), f_{1}(v)\right)=\operatorname{gcd}\left(f_{1}\left(v_{i}\right), f_{1}\left(v_{j}\right)\right)=$ $\operatorname{gcd}\left(f\left(v_{i}\right), f\left(v_{j}\right)\right)=1$ as $v_{i}$ and $v_{j}$ are adjacent vertices in the prime graph $G$ with the prime labelling $f$. Thus in all the possibilities $f_{1}$ admits a prime labeling for $G_{1}$. Hence $G_{1}$ is a prime graph.

Illustration 2.5: In the following Figures 3 and 4 prime labeling of a graph $G$ of order 5 and the prime labeling for the graph $G_{1}$ obtained by identifying the vertices of $G$ with label 1 and 5 are shown.

Theorem 2.6: The graph obtained by identifying any two vertices of $P_{n}$ is a prime graph.

Proof: Let $v_{1}, v_{2}, \cdots, v_{n}$ be the vertices of $P_{n}$. Let $u$ be the new vertex of the graph $G$ obtained by identifying two distinct vertices $v_{a}$ and $v_{b}$ of $P_{n}$. Then $G$ is nothing but a cycle (possibly loop) with at the most two

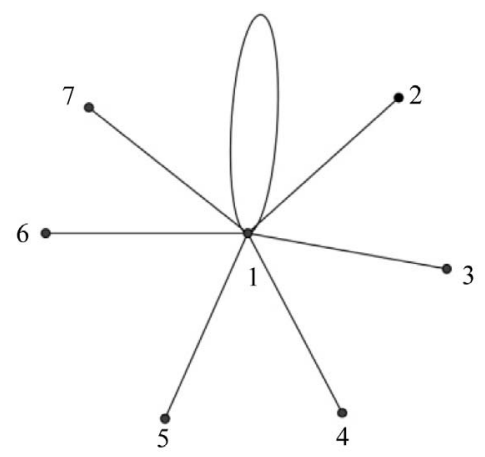

Figure 1. The prime labeling of the graph obtained by identifying the apex vertex with a pendant vertex in $K_{1,7}$.

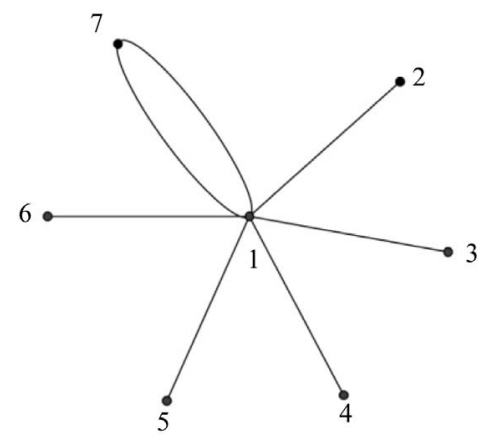

Figure 2. The prime labeling of the graph obtained by identifying two of the pendant vertices in $K_{1,7}$.

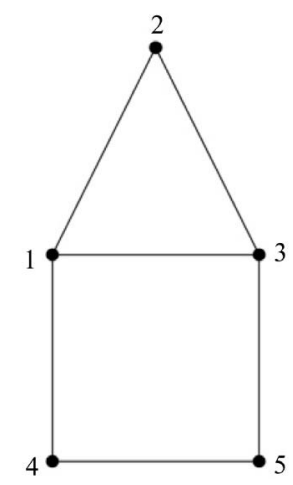

Figure 3. The prime labeling of a graph of order five.

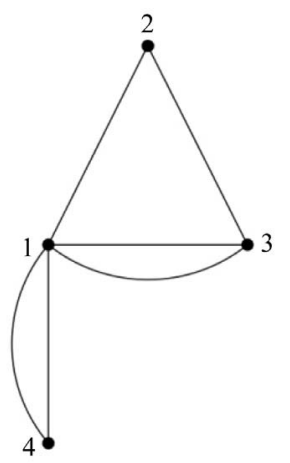

Figure 4. The prime labeling of the graph obtained by identifying the vertices of Figure 3 with label 1 and 5. 
paths attached at $u$. Such graph is a prime graph as proved in Vaidya and Prajapati [10].

Illustration 2.7: In the following Figures 5-9 prime labelings for $P_{5}$ and the graphs obtained by identifying two vertices in various possible ways are shown.

\section{Strongly Prime Graphs}

Definition 3.1: A graph $G$ is said to be a strongly prime

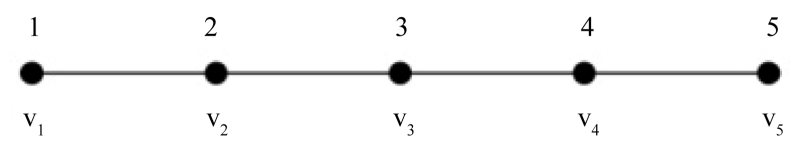

Figure 5. A prime labeling of $\boldsymbol{P}_{5}$.

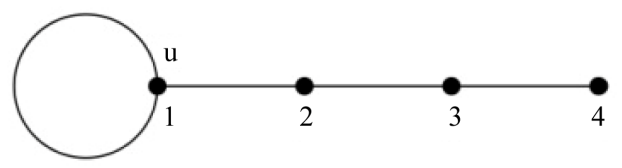

Figure 6. A prime labeling of the graph obtained by identifying $v_{1}$ and $v_{2}$ of $P_{5}$ of Figure 5 .

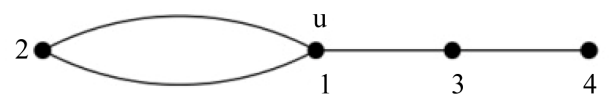

Figure 7. A prime labeling of the graph obtained by identifying $v_{1}$ and $v_{3}$ of $P_{5}$ of Figure 5 .

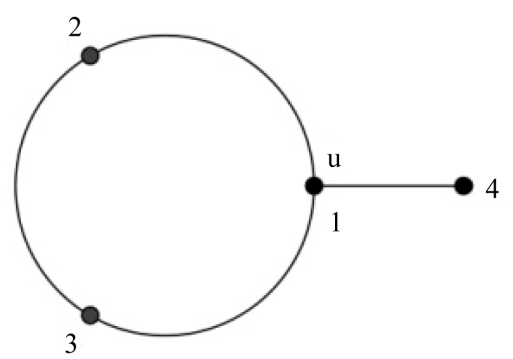

Figure 8. A prime labeling of the graph obtained by identifying $v_{1}$ and $v_{4}$ of $P_{5}$ of Figure 5 .

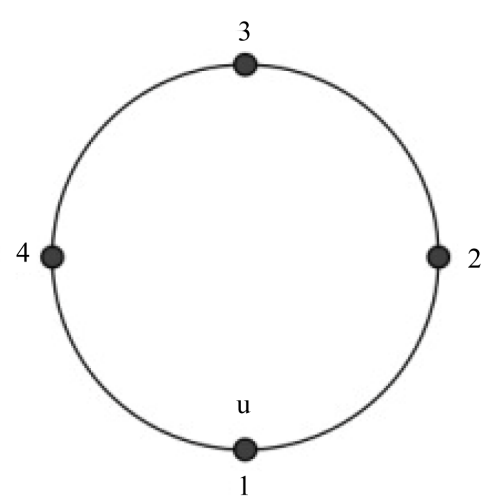

Figure 9. A prime labeling of the graph obtained by identifying $v_{1}$ and $v_{5}$ of $P_{5}$ of Figure 5. graph if for any vertex $v$ of $G$ there exits a prime labeling $f$ satisfying $f(v)=1$.

Observation 3.2: $K_{3}$ is a strongly prime graph as any vertex of $K_{3}$ can be assigned label 1 and the remaining vertices can be assigned label 2 and 3 as shown in Figure 10.

Observation 3.3: If $e$ is an edge of $K_{4}$ then $G=K_{4}-e$ is a prime graph (see Figure 11) but it is not a strongly prime graph. If possible either of the vertices of $G$ with degree 2 can be assigned the label 1 . Suppose $v_{1}$ is assigned the label 1 then available vertex labels for the remaining three vertices of $G-v_{1}$ are 2, 3 and 4 . Consequently the vertices corresponding to the labels 2 and 4 are adjacent in $G-v_{1}$. See Figure 12.

Observation 3.4: Every spanning subgraph of a strongly prime graph is a strongly prime graph. Because every spanning subgraph of a prime graph is a prime graph as proved by Seoud and Youssef [11].

Theorem 3.5: Every path is a strongly prime graph.

Proof: Let $v_{1}, v_{2}, \cdots, v_{n}$ be the consecutive vertices of $P_{n}$. If $v_{a}$ is any arbitrary vertex of $P_{n}$ then we have the following possibilities:

Case 1: If $v_{a}$ is either of the pendant vertices (say $\left.v_{a}=v_{1}\right)$ then the function $f: V\left(P_{n}\right) \rightarrow\{1,2, \cdots, n\}$ defined by $f\left(v_{i}\right)=i$, for all $i=1,2, \cdots, n$, is a prime labeling for $P_{n}$ with $f\left(v_{a}\right)=f\left(v_{1}\right)=1$.

Case 2: If $v_{a}$ is not a pendant vertex then $a=j$ for some $j \in\{2,3, \cdots, n-1\}$ then the function $f: V\left(P_{n}\right) \rightarrow\{1,2, \cdots, n\}$ defined by

$$
f\left(v_{i}\right)= \begin{cases}n+i-j+1 & \text { if } i=1,2, \cdots, j-1 ; \\ i-j+1 & \text { if } i=j, j+1, \cdots, n,\end{cases}
$$

is a prime labeling with $f\left(v_{a}\right)=f\left(v_{j}\right)=1$.

Thus from the cases described above $P_{n}$ is a strongly prime graph.

Illustration 3.6: It is possible to assign label 1 to arbitrary vertex of $P_{5}$ in order to obtain different prime labeling as shown in Figures 13-18.

Theorem 3.7: Every cycle is a strongly prime graph.

Proof: Let $v_{1}, v_{2}, \cdots, v_{n}$ be the consecutive vertices of $C_{n}$. Let $v$ be an arbitrary vertex of $C_{n}$. Then $v=v_{j}$ for some $j \in\{1,2, \cdots, n\}$. The function $f: V\left(C_{n}\right) \rightarrow\{1,2, \cdots, n\}$ defined by

$$
f\left(v_{i}\right)= \begin{cases}n+i+1-j & \text { if } i=1,2, \cdots, j-1 ; \\ i-j+1 & \text { if } i=j, j+1, \cdots, n,\end{cases}
$$

is a prime labeling for $C_{n}$ with $f(v)=f\left(v_{j}\right)=1$. Thus $f$ admits prime labeling as well as it is possible to assign label 1 to any arbitrary vertex of $C_{n}$. That is $C_{n}$, is strongly prime graph.

Theorem 3.8: $K_{1, n}$ is a strongly prime graph.

Proof: For $n=1,2$ the respective graphs $P_{2}$ and $P_{3}$ are strongly prime graphs as proved in the Theorem 3.5. 


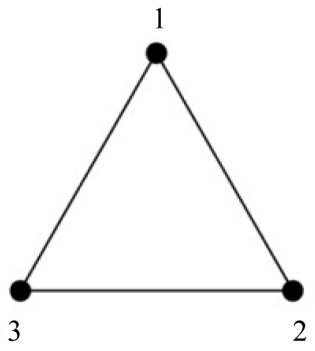

Figure 10. A strongly prime labeling of $K_{3}$.

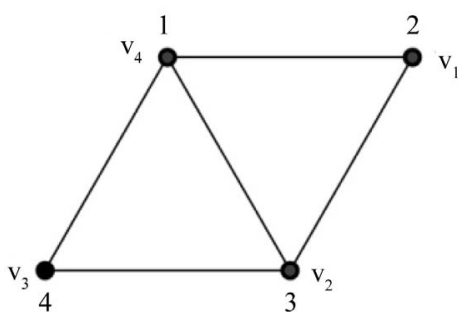

Figure 11. A prime labeling of $K_{4}-e$.

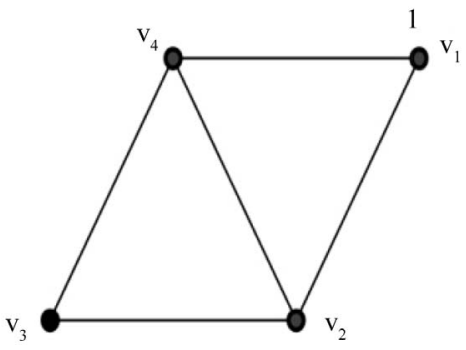

Figure 12. $K_{4}-e$ is not a strongly prime graph.

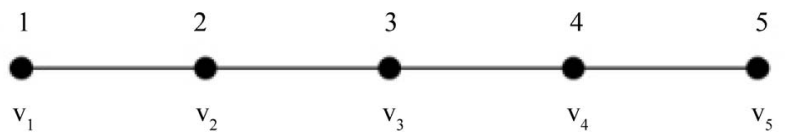

Figure 13. A prime labeling of $P_{5}$ having $v_{1}$ as label 1.

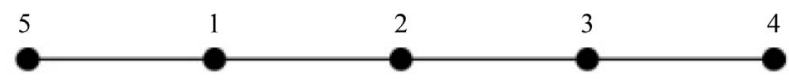

Figure 14. A prime labeling of $P_{5}$ having $v_{2}$ as label 1.

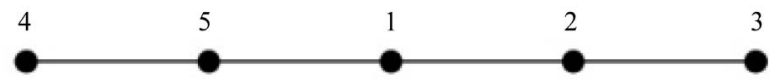

Figure 15. A prime labeling of $P_{5}$ having $v_{3}$ as label 1.

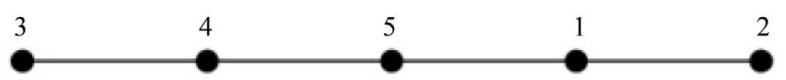

Figure 16. A prime labeling of $P_{5}$ having $v_{4}$ as label 1 .

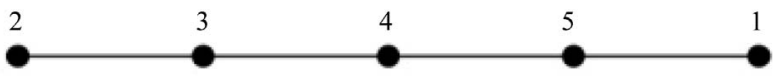

Figure 17. A prime labeling of $P_{5}$ having $v_{5}$ as label 1 .

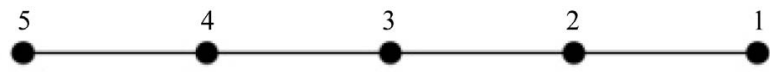

Figure 18. A prime labeling of $P_{5}$ having $v_{5}$ as label 1.

For $n>2$ let $v_{0}$ be the apex vertex and $v_{1}, v_{2}, \cdots, v_{n}$ be the pendant vertices of $K_{1, n}$.

If $x$ is any arbitrary vertex of $K_{1, n}$ then we have the following possibilities:

Case 1: If $x$ is the apex vertex then $x=v_{0}$. Then the function $f: V\left(K_{1, n}\right) \rightarrow\{1,2, \cdots, n+1\}$ defined by $f\left(v_{i}\right)=i+1$ for $i=0,1,2, \cdots, n$ is a prime labeling on $K_{1, n}$ with $f(x)=f\left(v_{0}\right)=1$.

Case 2: If $x$ is one of the pendant vertices then $x=v_{j}$ for some $j \in\{1,2, \cdots, n\}$. Define

$f: V \rightarrow\{1,2, \cdots, n, n+1\}, f\left(v_{0}\right)=p$, where $p$ is the largest prime less than or equal to $n$ and the remaining $n-1$ vertices are distinctly labeled from

$\{2, \cdots, n, n+1\}-\{p\}$. According to Bertrand's postulate $\left\lfloor\frac{n+1}{2}\right\rfloor<p<n+1$. Therefore $p$ is co-prime to every integer from $\{1,2, \cdots, n, n+1\}-\{p\}$. Thus every edge $e=a b$ is incident to the apex vertex $v_{0}$ whose label is $p$, thus $\operatorname{gcd}(f(a), f(b))=\operatorname{gcd}(p, f(b))=1$ or $\operatorname{gcd}(f(a), f(b))=\operatorname{gcd}(f(a), p)=1$. Hence this function $f$ admits a prime labeling on $K_{1, n}$ with $f(x)=f\left(v_{j}\right)=1$.

Thus from all the cases described above $K_{1, n}$ is a strongly prime graph.

Illustration 3.9: It is possible to assign label 1 to arbitrary vertex of $K_{1,7}$ in order to obtain prime labeling as shown in Figures 19 and 20.

Theorem 3.10: $W_{n}$ is a strongly prime graph for every even positive integer $n \geq 4$.

Proof: Let $v_{0}$ be the apex vertex and $v_{1}, v_{2}, \cdots, v_{n}$ be the consecutive rim vertices of $W_{n}$. Let $x$ be an arbitrary vertex of $W_{n}$. We have the following possibilities:

Case 1: $x$ is the apex vertex of $W_{n}$ that is $x=v_{0}$. Then the function $f: V\left(W_{n}\right) \rightarrow\{1,2, \cdots, n+1\}$ defined as

$$
f\left(v_{i}\right)=i+1, i=0,1,2, \cdots, n .
$$

Obviously $f$ is an injection. For an arbitrary edge $e=a b$ of $G$ we claim that $\operatorname{gcd}(f(a), f(b))=1$. To prove our claim the following subcases are to be considered.

Subcase (1): if $e=v_{i} v_{i+1}$ for some $i \in\{1,2, \cdots, n-1\}$ 


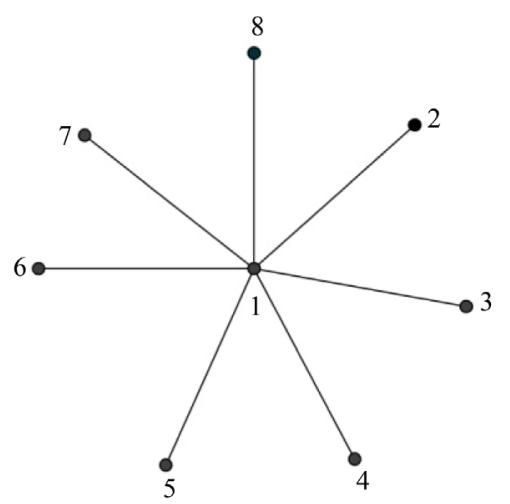

Figure 19. A prime labeling of $K_{1,7}$ with the apex vertex as label 1.

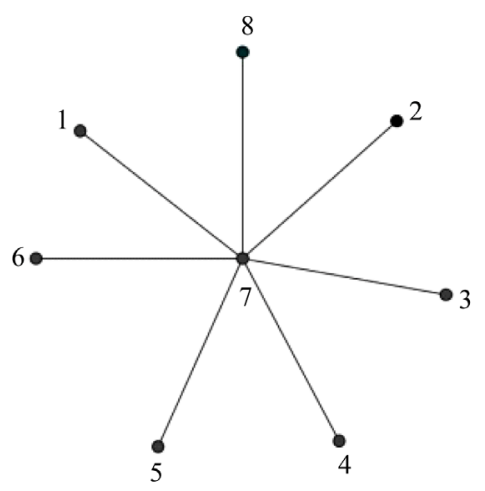

Figure 20. A prime labeling of $K_{1,7}$ with a pendant vertex as label 1.

then $\operatorname{gcd}\left(f\left(v_{i}\right), f\left(v_{i+1}\right)\right)=\operatorname{gcd}(i+1, i+1+1)$

$=\operatorname{gcd}(i+1, i+2)=1$ as $i+1$ and $i+2$ are consecutive positive integers.

Subcase (2): if $e=v_{n} v_{1}$ then $\operatorname{gcd}\left(f\left(v_{n}\right), f\left(v_{1}\right)\right)=$ $\operatorname{gcd}(n+1,2)=1$ as $n+1$ is an odd integer and it is not divisible by 2 .

Subcase (3): if $e=v_{0} v_{i}$ for some $i \in\{1,2, \cdots, n\}$ then $\operatorname{gcd}\left(f\left(v_{0}\right), f\left(v_{i}\right)\right)=\operatorname{gcd}(1, i+1)=1$.

Case 2: $x$ is one of the rim vertices. We may assume that $x=v_{p-1}$ where $p$ is the largest prime less than or equal to $n+1$. According to the Bertrand's Postulate such a prime $p$ exists with $\left\lfloor\frac{n+1}{2}\right\rfloor<p<n+1$. Define a function $f: V\left(W_{n}\right) \rightarrow\{1,2,3, \cdots, n+1\}$ as

$$
f\left(v_{i}\right)= \begin{cases}p, & \text { if } i=0 ; \\ i+1, & \text { if } i \in\{1,2, \cdots, n\}-\{p-1\} ; \\ 1, & \text { if } i=p-1 .\end{cases}
$$

The only difference between the definition of labeling functions of (1) and (2) is the labels 1 and $p$ are interchanged. Then clearly $f$ is an injection.

For an arbitrary edge $e=a b$ of $G$ we claim that $\operatorname{gcd}(f(a), f(b))=1$. To prove our claim the following subcases are to be considered.

Subcase (2): If $e=v_{0} v_{i}$ for some $i \in\{1,2, \cdots, n\}$ then $\operatorname{gcd}\left(f\left(v_{0}\right), f\left(v_{i}\right)\right)=\operatorname{gcd}\left(p, f\left(v_{i}\right)\right)=1$ as $p$ is co-prime to every integer from $\{1,2, \cdots, n+1\}-\{p\}$.

Subcase (2): If $e=v_{i} v_{i+1}$ for some $i \in\{1,2, \cdots, p-3\} \cup\{p, p+1, \cdots, n\}$ then $\operatorname{gcd}\left(f\left(v_{i}\right), f\left(v_{i+1}\right)\right)=\operatorname{gcd}(i+1, i+2)=1$ as $i+1$ and $i+2$ are consecutive positive integers.

Subcase (3): If $e=v_{i} v_{i+1}$ for $i=p-2$ then $\operatorname{gcd}\left(f\left(v_{i}\right), f\left(v_{i+1}\right)\right)=\operatorname{gcd}\left(f\left(v_{p-2}\right), f\left(v_{p-1}\right)\right)=$ $\operatorname{gcd}(p-1,1)=1$.

Subcase (4): If $e=v_{i} v_{i+1}$ for $i=p-1$ then $\operatorname{gcd}\left(f\left(v_{i}\right), f\left(v_{i+1}\right)\right)=\operatorname{gcd}\left(f\left(v_{p-1}\right), f\left(v_{p}\right)\right)=$ $\operatorname{gcd}(1, p+1)=1$

Thus in all the possibilities described above $f$ admits prime labeling as well as it is possible to assign label 1 to any arbitrary vertex of $W_{n}$. That is, $W_{n}$ is a strongly prime graph for every even positive integer $n \geq 4$.

Illustration 3.11: It is possible to assign label 1 to arbitrary vertex of $W_{8}$ in order to obtain prime labeling as shown in Figures 21 and 22.

Corollary 3.12: The friendship graph $C_{3}^{(n)}$ is a strongly prime graph.

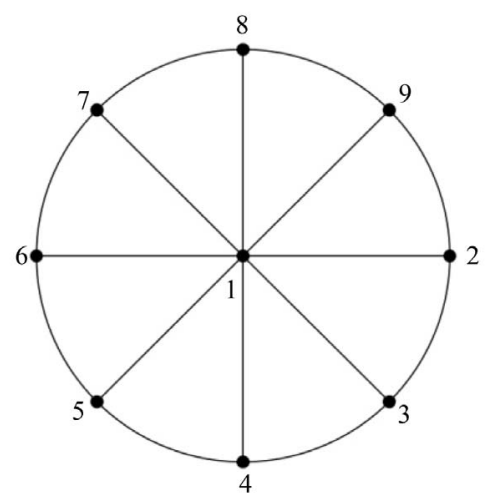

Figure 21. A prime labeling of $W_{8}$ with the apex vertex as label 1.

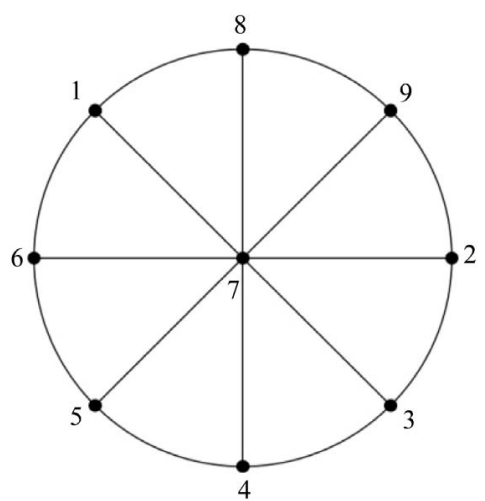

Figure 22. A prime labeling of $W_{8}$ with a rim vertex as label 1. 
Proof: The friendship graph $C_{3}^{(n)}$ is a one point union of $n$ copies of $C_{3}$. It can also be thought as a graph obtained by deleting every alternate rim edge of $W_{2 n}$. Being a spanning subgraph of strongly prime graph $W_{2 n}, C_{3}^{(n)}$ is a strongly prime graph.

Corollary 3.13: The star $K_{1,2 n}$ is a strongly prime graph.

Proof: $K_{1,2 n}$ is obtained from strongly prime graph $W_{2 n}$ by deleting all the rim edges of the $W_{2 n}$. Being a spanning subgraph of strongly prime graph $W_{2 n}, K_{1,2 n}$ is a strongly prime graph.

\section{Concluding Remarks}

The prime numbers and their behaviour are of great importance as prime numbers are scattered and there are arbitrarily large gaps in the sequence of prime numbers. If these characteristics are studied in the frame work of graph theory then it is more challenging and exciting as well.

Here we investigate several results on prime graphs. This discussion becomes more interesting in the situation when two vertices of a graph are identified. We also introduce a concept of strongly prime graph. As every prime graph is not a strongly prime graph it is very exciting to investigate graph families which are strongly prime graphs. We investigate several classes of prime graph which are strongly prime graph.

\section{Acknowledgements}

The authors are highly thankful to the anonymous referee for valuable comments and constructive suggestions.

\section{REFERENCES}

[1] J. Gross and J. Yellen, "Graph Theory and Its Applica- tions," CRC Press, Boca Raton, 1999.

[2] D. M. Burton, "Elementary Number Theory," 2nd Edition, Brown Publishers, New York, 1990.

[3] J. A. Gallian, "A Dynamic Survey of Graph Labeling," The Electronic Journal of Combinatorics, Vol. 18, 2011. http://www.combinatorics.org/Surveys/ds6.pdf

[4] A. Tout, A. N. Dabboucy and K. Howalla, "Prime Labeling of Graphs," National Academy Science Letters, Vol. 11, 1982, pp. 365-368.

[5] H. L. Fu and K. C. Huang, "On Prime Labellings," Discrete Mathematics, Vol. 127, No. 1-3, 1994, pp. 181-186. doi:10.1016/0012-365X(92)00477-9

[6] S. M. Lee, I. Wui and J. Yeh, "On the Amalgamation of Prime Graphs," Bulletin of the Malaysian Mathematical Sciences Society (Second Series), Vol. 11, 1988, pp. 59-67.

[7] T. Deretsky, S. M. Lee and J. Mitchem, "On Vertex Prime Labelings of Graphs," In: J. Alvi, G. Chartrand, O. Oellerman, A. Schwenk, Eds., Graph Theory, Combinatorics and Applications: Proceedings of the 6th International Conference Theory and Applications of Graphs, Wiley, New York, 1991, pp. 359-369.

[8] S. K. Vaidya and K. K. Kanani, "Prime Labeling for Some Cycle Related Graphs," Journal of Mathematics Research, Vol. 2, No. 2, 2010, pp. 98-103. http://ccsenet.org/journal/index.php/jmr/article/view/4423/ 4743

[9] S. K. Vaidya and U. M. Prajapati, "Some Switching Invariant Prime Graphs," Open Journal of Discrete Mathematics, Vol. 2, No. 1, 2012, pp. 17-20. doi:10.4236/ojdm.2012.21004

[10] S. K. Vaidya and U. M. Prajapati, "Some Results on Prime and k-Prime Labeling," Journal of Mathematics Research, Vol. 3, No. 1, 2011, pp. 66-75. http://ccsenet.org/journal/index.php/jmr/article/download/ $7881 / 6696$

[11] M. A. Seoud and M. Z. Youssef, "On Prime Labeling of Graphs," Congressus Numerantium, Vol. 141, 1999, pp. 203-215. 